\title{
A Numerical Approach to the Solution of Radio Diffraction Problems
}

\author{
J. K. Hargreaves ${ }^{1}$ and S. Hargreaves ${ }^{2}$
}

(Received June 5, 1963)

\begin{abstract}
It is proposed that some diffraction problems can be conveniently solved by a direct numerical integration of the Fresnel-Kirchhoff formula. The required properties of the diffraction screen are represented by a series of numbers which can be either regular and periodic, or partially random. The necessary limits and integration intervals are considered, and the method is found to be convenient for Fresnel diffraction and for irregularities not too large compared to the wavelength. Both deep and shallow modulation can be treated. The accuracy of the computations is verified in a simple case of sinusoidal modulation, and some new results are derived for random phase screens.
\end{abstract}

\section{Introduction}

Diffraction is an important phenomenon of radio propagation, which is likely to be encountered in the many instances of irregular propagation media. In theoretical treatments it is usually assumed that the wave emerges from the irregular medium with random fluctuations of amplitude and/or phase along the wave front, and that the diffraction problem concerns just the evolution of this initial modulation as the wave propagates, no additional modulation being introduced further on. This leads to the concept of a thin diffraction "screen" where all fluctuations of signal are supposed to be introduced. Given the properties of the diffraction screen it is then required to find the properties of the signal over an observation plane some distance beyond it. The computed diffraction pattern may subsequently be compared with experimental data, in order to make deductions about the character of the irregular medium.

Even in this simplified form the problem is not easy. The greatest step towards its solution was the development of the "angular spectrum" theory [Booker, Ratcliffe, and Shinn, 1950; Ratcliffe, 1956] which, drawing analogy with the frequency spectrum of a time-modulated signal, provided a valuable physical concept of the process and leads to direct answers in the more simple instances - for example, when the diffraction screen introduces only "shallow" modulation and the observation plane is far away from it. More difficult are the situations where the observation plane is relatively close to the screeni.e., Fresnel diffraction - or when the screen introduces "deep" modulation, which is usually taken to imply phase fluctuations exceeding one radian or amplitude fluctuations exceeding one neper. Hewish [1951], Bowhill [1957, 1961], and Wagner [1962] have attacked aspects of these problems by the angular spectrum method. An alternative approach, involv-

1 Now at the National Bureau of Standards, Boulder, Colo.

2 Now at National Center for Atmospheric Research, Boulder, Colo. ing direct application of Huygen's principle through the Fresnel-Kirchhoff formula, has been taken by Mercier [1959, 1962].

By dint of mathematical sophistication both methods have yielded valuable results; yet simplifying assumptions, such as of a Gaussian distribution of fluctuations, or a small-angle approximation, have normally had to be invoked. Moreover, it does not seem to have been possible to obtain any numerical results for random screens whose phase modulation exceeds an rms value of $\sqrt{2}$ radians. The approach to be offered here, on the other hand, is almost entirely numerical, being based on a simple numerical integration of the Fresnel-Kirchhoff formula. The properties of random screens are simulated by the generation of correlated random numbers. While the mathematics are now elementary, a high-speed computer and appropriate programming skill are required. The merits of an analytical solution are lost, but it seems that the following advantages may be gained by the numerical approach:

1. There is no need to restrict the screen properties for mathematical convenience. Any form of screen may be assumed, and the appropriate numerical solution will be obtained in a form suitable for direct comparison with experimental data.

2. The Fresnel, or small-angle, approximations are avoided.

3. Deeply modulated screens do not present any particular difficulty.

Thus, the lost convenience of an analytical answer may conceivably be compensated by the ability to solve a greater range of problems.

The present task is to explore the possibilities of the numerical method. We shall try to establish confidence in its accuracy by working out some simple cases whose answers are known from previous work. We will examine the limits within which the method looks practicable (these are in effect limits of computing speed) and compute some more difficult instances as examples. 


\section{Formulation}

The starting point is the Fresnel-Kirchhoff diffraction formula ${ }^{3}$ [Born and Wolf, 1959], which says that the field at $R$ due to a source $S$ is

$$
U=-\frac{j}{2 \lambda} \int_{A} \frac{\exp j k(r+s)}{r s}\left(\cos \chi_{r}-\cos \chi_{s}\right) d A
$$

where

$d A$ is an element of a surface $A$ enclosing $R$ or $S$ (or an infinite plane between them), $r$ and $s$ are the distances of $R$ and $S$ from $d A$, $\chi_{r}$ and $\chi_{s}$ are the angles between the normal to $d A$ and the directions to $R$ and $S$,

and

$$
k=\frac{2 \pi}{\lambda} \text {, for wavelength } \lambda .
$$

If the source is at infinity,

$$
U=-\frac{j}{2 \lambda} \int_{A} \frac{\exp j k r}{r}\left(1+\cos \chi_{r}\right) d A
$$

In the present work, $A$ will be a plane distant $h$ from $R$, as in figure 1 , and the simplifying assumption will be made that irregularities exist in the $x$-direction only, being infinitely elongated in the z-direction. Diffraction in the z-direction does not then concern us, and the formulation can be reduced to two dimensions by applying to the $z$-direction the usual Fresnel approximations:

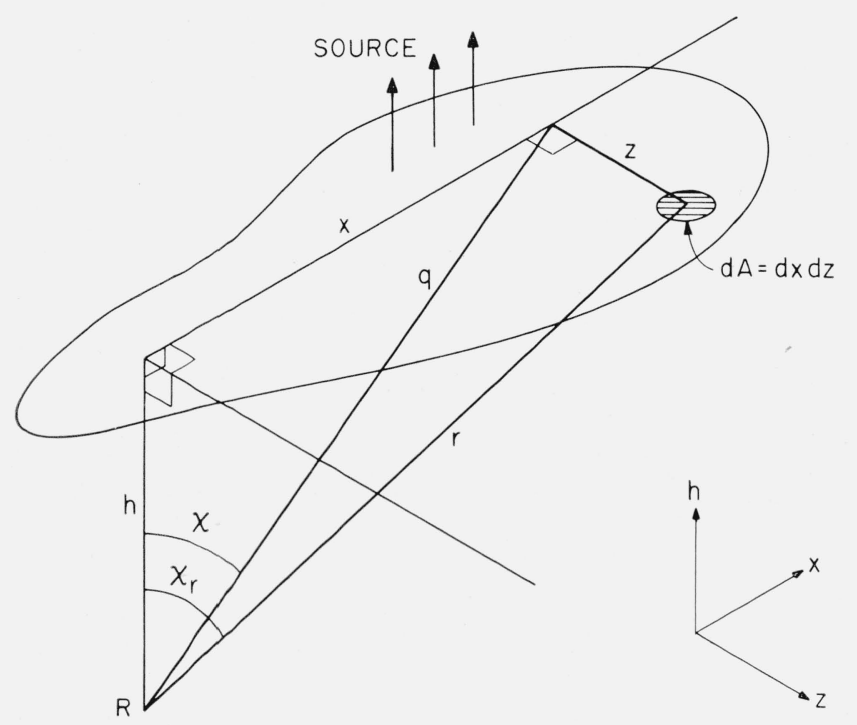

Figure 1. Geometry of the model.

${ }^{3}$ Although not rigorous, the Fresnel-Kirchhoff formula is quite the most tractable expression of Huygen's principle. The usual objection that it neglects boundary effects at an opaque screen will not apply here because no discontinuities will be involved, and curvature of the constant-phase front will always be greater than a wavelength. The derivation of (1) from the more basic HelmholtzKirchhoff equation assumes $r, s,>>\lambda / 2 \pi$. $r \approx q\left(1+\frac{z^{2}}{2 q^{2}}\right)$ in the exponent,

and

$$
r \approx q \text { in the denominator, }
$$

$$
\chi_{r} \approx \chi .
$$

Whence, for the model in figure 2,

$$
U=\sqrt{\frac{1}{j \lambda}} \int_{x=-\infty}^{+\infty} \frac{\exp j k q}{q^{\frac{1}{2}}} \frac{(1+\cos \chi)}{2} d x .
$$

In contrast to previous analytical solutions, no approximation has been made in the $x$-direction where the irregularities are.

It is now supposed that the constant-phase front of a wave which originated at infinity has been delayed during propagation to the plane $A$ by amounts $\Delta h(x)$, as in figure 2. 'Thus, assuming' $\Delta h \ll h$,

$$
U=\sqrt{\frac{1}{j \lambda}} \int_{x=-\infty}^{+\infty} \frac{\exp j k\left(\sqrt{h^{2}+x^{2}}+\Delta h \cos \chi\right)}{\sqrt{q}} \cdot \frac{(1+\cos \chi)}{2} d x
$$

Replacing this by a summation, and expressing distances in terms of the wavelength, gives

$$
U=\frac{\Delta X}{\sqrt{j H}} \sum_{-X_{L}}^{+X_{L}} \frac{1+S e}{2 S e^{3 / 2}} \exp 2 \pi j[H S e+\Delta H / S e]
$$

where

$$
\Delta X=\frac{\Delta x}{\lambda}, \Delta H=\frac{\Delta h}{\lambda}, H=\frac{h}{\lambda}, \text { and } S e=S e c \chi=\left(1+\frac{x^{2}}{h^{2}}\right)^{\frac{1}{2}} .
$$

The limits are now $\pm X_{L}$ instead of $\pm \infty$. For the other case of practical interest, where both $S$ and $R$ are distant $h$ from the screen, similar reasoning gives

$$
U=\frac{\Delta X}{\sqrt{2 j H h}} \sum_{-X_{L}}^{+X_{L}} \frac{\exp 2 \pi j\left[2 H S e+\frac{\Delta H}{S e}\right]}{S e^{5 / 2}} .
$$

In general it will be convenient to express the field as an amplitude and a phase term:

$$
U=M \exp (j \varphi) \text {. }
$$

Our general procedure will be as follows. The modulation function $\Delta h(x)$ is generated, and the

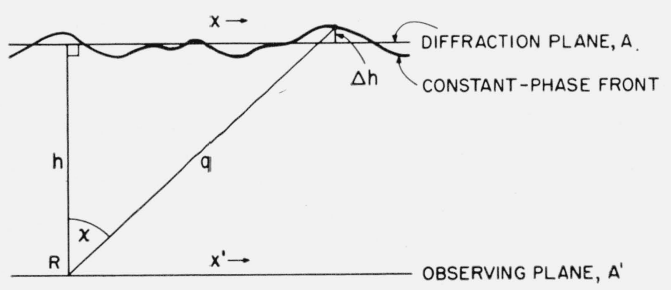

Figure 2. Phase irregularities. 
field $U$ is determined from (5) or (6). The modulation function is then translated along the $x$-direction in steps of $\Delta x^{\prime}$, the corresponding $U\left(x^{\prime}\right)$ being evaluated at each step. Thus the diffracted field along the observing plane will be derived, given an assumed initial modulation at plane $A$. In the present work only sinusoidal or random screens will be used, and the diffracted fields will be derived from (5), corresponding to the source at infinity.

\section{Preliminary Tests: Unperturbed Wave Front}

Before (5) can be used in computing diffraction problems, it is necessary to find the smallest summation limit, $X_{L}$, and the largest increment, $\Delta X$, that can be used without undue error. They in turn specify how many terms, $\frac{\left(2 X_{L}+1\right)}{\Delta X}$, must be taken, and so indicate the feasibility of obtaining solutions with a given computer. For this reason initial computations were made assuming the wavefront to be unperturbed-i.e., $\Delta H=0$ - in which case the result should be $M=1, \varphi=0$.

The progressive summation of (5) may be regarded as the construction of a spiral. When $X$ is large relative to $H$ the terms become small and equal to $\frac{\Delta X}{2 \sqrt{X}}$ in magnitude. Provided $\Delta X$ is small, one revolution of the spiral is completed every time $X$ increases by unity, i.e., every $\frac{1}{\Delta X}$ terms, and the spiral has radius $\frac{1}{4 \pi \sqrt{X}}$. Thus, if (5) is summed to $\pm X_{L}$, where $X_{L}$ is large relative to $H$, there is a residual error with magnitude

$$
\epsilon=\frac{1}{2 \pi \sqrt{X_{L}}},
$$

with respect to the sum to infinity. In the more general case when $X_{L}$ is not necessarily large compared to $H$ and the individual terms are of magnitude

$$
\frac{\Delta X}{2 \sqrt{H}} \cdot \frac{(1+S e)}{S e^{3 / 2}},
$$

the residual error may be obtained from figure 3 which gives $\epsilon \sqrt{\frac{H}{5}}$ as a function of $\frac{X_{L}}{H}$. This was computed for $H=5$ and shows, for instance, that $\epsilon=2.6$ percent if $\frac{X_{L}}{H}=9$ and $H=5$. In this instance it would be necessary to include ${ }_{\Delta X}^{91}$ terms. Fewer terms would be needed in the summation of (6) to the same accuracy because the individual terms, again at large $X$, fall off in magnitude as $1 / X^{5 / 2}$ rather than $1 / X^{1 / 2}$.

The procedure adopted for computation with (5) is to evaluate the series up to some $X_{L}$ for which $\epsilon$ is

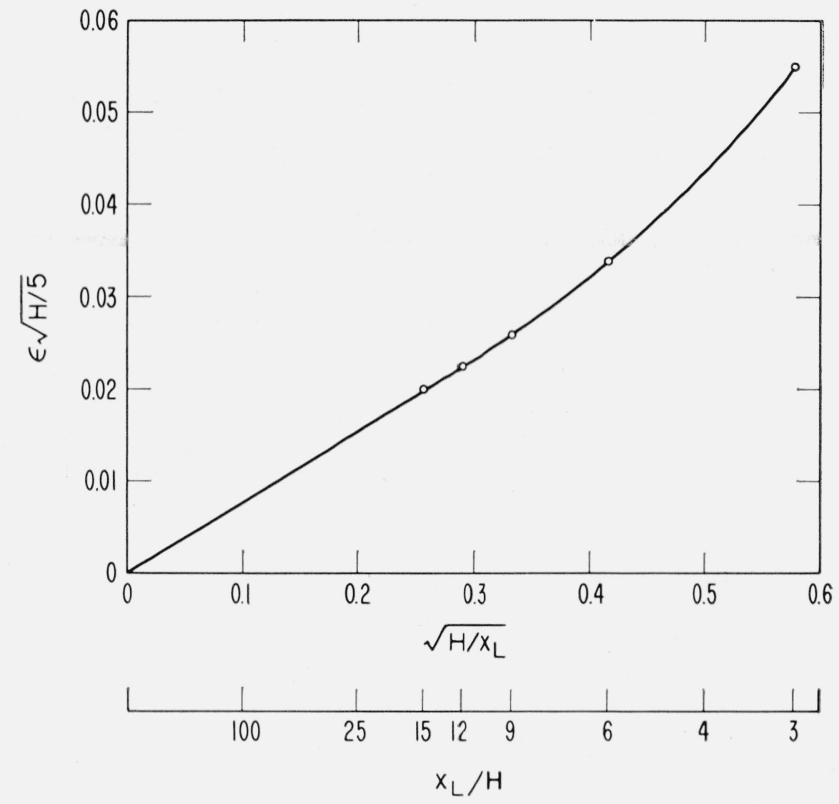

Figure 3. $\epsilon \sqrt{\mathrm{H} / 5}$ in terms of $\mathrm{X}_{\mathrm{L}} / \mathrm{H}$.

suitably small (usually 2 to $3 \%$ ) and then to add on a computed error term of appropriate amplitude and phase. Test computations with $H$ between $2 \frac{1}{2}$ and 20 , taking $\epsilon=2.5$ percent, gave remaining errors of less than 0.2 percent after the addition of the error term.

In the course of the foregoing tests it was found that the increment, $\Delta X$, had no significant effect on the results provided $\Delta X \leq 1 / 4$. It seems that a relatively large interval will suffice for an unmodulated wavefront. However, this may not hold for modulated waves, where it will obviously be necessary to delineate the perturbations in sufficient detail, and the question of increment will be considered again later.

\section{Sinusoidal Screens}

\subsection{Shallow Modulation}

Diffraction by a sinusoidal phase screen can readily be evaluated by the angular spectrum method [Hewish, 1951; Ratcliffe, 1956] when the modulation is shallow-i.e., when the fluctuations are small relative to 1 radian-and only first-order sidewaves need to be taken into account. It is therefore a suitable test of the numerical method. The modulation is of the general form

$$
\Delta H=\Delta H_{0} \cos \frac{2 \pi X}{P} .
$$

This calculation has been carried out by both the angular-spectrum and the numerical methods for a modulation depth $\left(\Delta H_{0}\right)$ of 0.01 wavelength and for different values of the period $(P)$, the distance from diffraction to observation plane $(H)$ being taken as 5 wavelengths. As is well known, the diffraction 
pattern is also sinusoidal, with the same period as the irregularities, but the actual magnitudes of the phase and amplitude fluctuations $(\hat{\varphi}$ and $\hat{M})$ depend on both $P$ and $H$. $\hat{\varphi}$ and $\hat{M}$ are shown as a function of $P$, for $H=5$, in figure 4 . The two methods, in fact, give virtually the same result, differences being no greater than $0.001 . X_{L} / H=9$ was used for the numerical method, and the same results were obtained for $\Delta X=1 / 8,1 / 16$, and $1 / 32$. The "total fluctuation," which is $\sqrt{\hat{\varphi}_{2}+\hat{M}^{2}}$, is independent of $P$ when $P$ is large compared to one wavelength, and figure 4 illustrates how the fluctuation alternates between amplitude and phase, being mainly in the phase for periods somewhat greater than the first Fresnel zone $(P>\sqrt{H})$.

The close agreement between the two methods in the case of a shallow sinusoidal phase screen shows that the numerical method is capable of good accuracy in a simple instance. We shall now evaluate some more complex examples, for which analytical results are less readily available.

\subsection{Deep Modulation}

As long as the fluctuations in the screen are small compared with 1 radian $\left(\Delta H_{0}<<1 / 2 \pi\right)$ the form of the diffraction pattern is independent of the initial fluctuation depth, and the values of $\hat{M}$ and $\hat{\varphi}$ are simply proportional to it. When the initial modulation approaches 1 radian in depth these simple truths fail and then the modulation can no longer be considered "shallow." The results of a series of computations for $\Delta H_{0}=0.1$ are plotted, suitably scaled, on figure 4 , and a considerable deviation from the form of the shallow modulation can be seen. In terms of the angular-spectrum concept, these changes with increasing depth of modulation are due to contributions from side-waves of higher

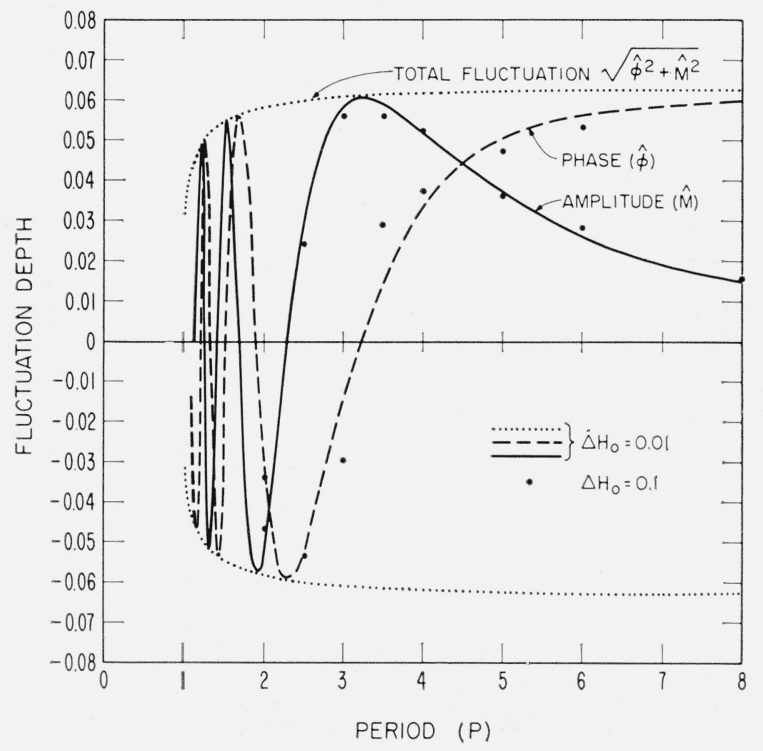

Figure 4. Shallow sinusoidal phase screen. order that introduce higher spacial harmonics into the diffraction pattern. Examples with $\Delta H_{0}$ near or exceeding $0.1-$ a phase fluctuation of $\pi / 5$ radianplainly cannot be treated as shallow modulations.

In the angular-spectrum method, problems invoking deep modulation require the addition of several side-waves whose amplitudes are specified by a Bessel function. The number of required terms increases rapidly with increasing modulation depth. By this technique Hewish [1951] computed diffraction patterns from initial phase modulations of up to 8 radians. In the present numerical method deeply modulated screens can be treated as easily as shallow ones, apart from the need to verify that the summation interval $(\Delta X)$ is adequately small. Figures 5 and 6 show the diffraction patterns computed for the conditions $H=5, P=3 \frac{1}{2}$, and $H=20$, $P=2$, with initial modulation depths $\left(\Delta \mathrm{H}_{0}\right)$ up to four wavelengths (25 radians). These show plainly the distortion of the sinusoidal form by increased modulation depth, with periodicities down to half a wavelength in evidence. In general the overall amplitude fluctuation of the diffracted signal does not change greatly beyond $\Delta H_{0}=0.1$; whereas the overall phase fluctuation (which has been plotted as a fraction of the input modulation $\varphi_{s}=2 \pi \Delta H_{0}$ ) tends to increase at least as far as $\Delta H_{0}=1.0$. The curves are similar in general appearance to those given by Hewish [1951], but a detailed comparison is not possible because he does not state his value of $P$.

\subsection{Summation Interval for Deep Modulation}

The foregoing computations were made with a summation interval $\Delta X=1 / 16$. To investigate the adequacy of this interval, some computations were repeated using other intervals, in the range from $1 / 32$ to unity. Figure 7 shows the patterns obtained in the case $H=20, P=2, \Delta H_{0}=0.1$. The results are identical from intervals $1 / 16$ to $1 / 4$, and even interval $1 / 2$ incurs very little error in spite of the fact that it barely specifies the waveform adequately. Computations for $H=5, P=3 \frac{1}{2}, \Delta H_{0}=1$, the most severe case of figure 5 , using $\Delta X=1 / 32,1 / 16,1 / 8$ and $1 / 4$, differed from each other by less than 0.0024 in either amplitude or phase; relative to a total fluctuation of 1.3 in amplitude and of 5.8 in phase this is a negligible error. An even more severe case in figure $6, H=20$, $P=2, \Delta H_{0}=4$, was computed using several intervals between $1 / 32$ and $1 / 8$. Here the results for $1 / 32$ and $1 / 16$ agree, yet those for $1 / 12$ and $1 / 8$ were utterly different, as figure 8 shows. It is curious that the error should increase so drastically for intervals which are only slightly too large. Figure 9 clearly illustrates the rapid deterioration at two particular points of the pattern: intervals less than 1/15 are accurate, 1/15 shows a small error, and intervals larger than 1/15 cause unacceptably large errors.

The sharpness of the transition between satisfactory and unsatisfactory intervals has one useful aspect. It is possible to test an interval by simply repeating the computation with the interval doubled. It the two results agree it is highly probable that 

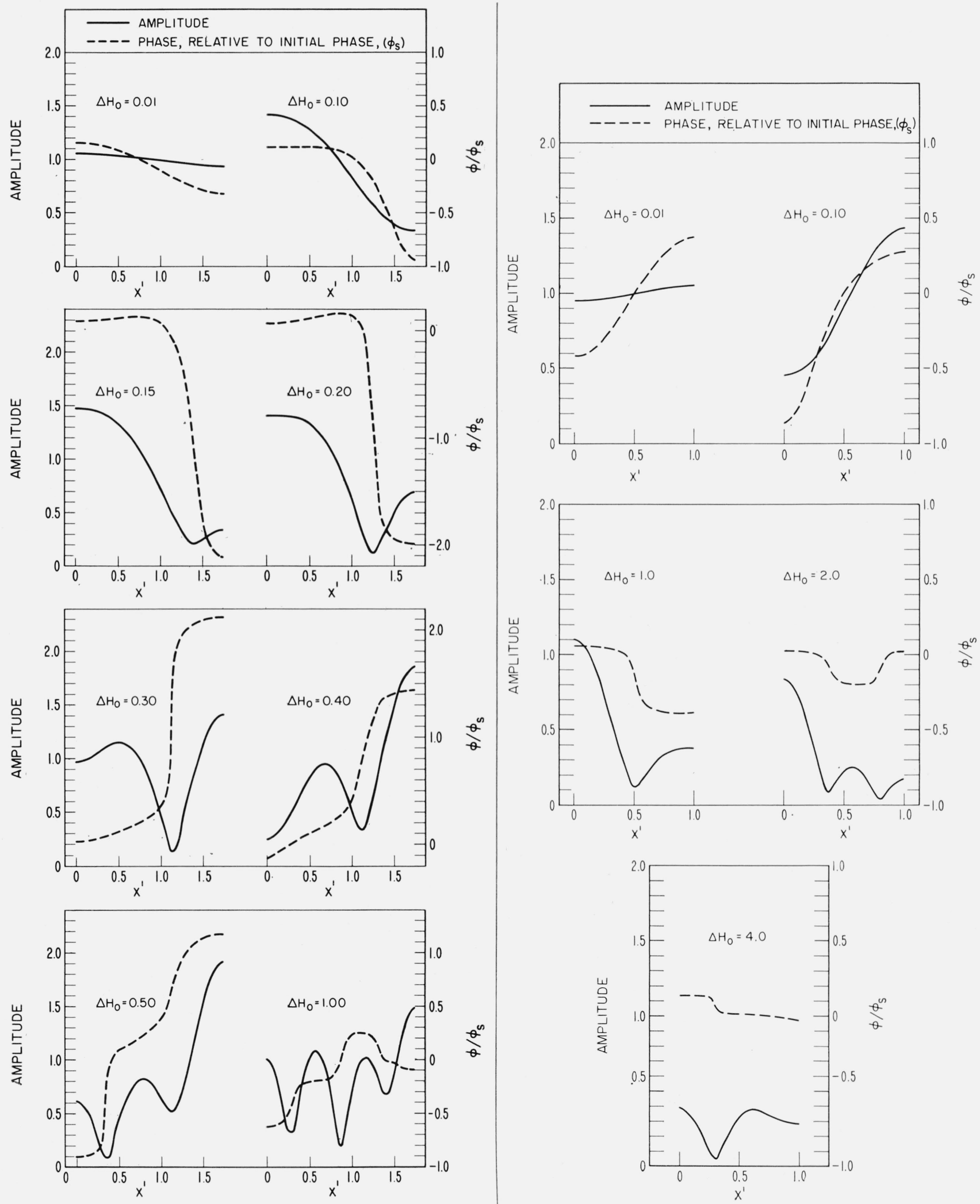

Figure 5. Deeply modulated sinusoidal phase screens: $\mathrm{P}=3 \frac{1}{1} 2$. Figure 6. Deeply modulated sinusoidal phase screens: $\mathrm{P}=\mathbf{2}$. 

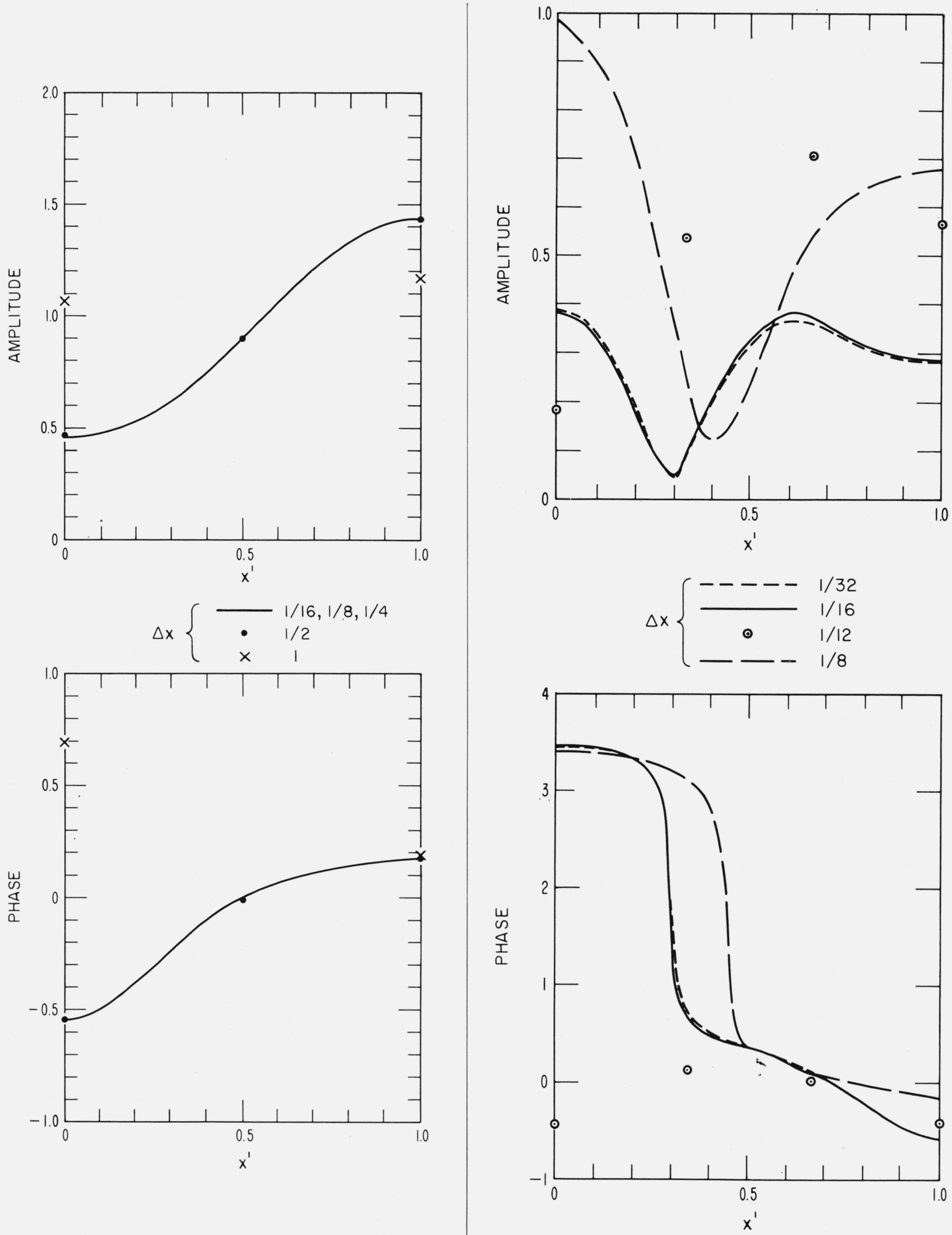

Figure 7. Effect of summation interval $(\Delta X)$ for $\Delta \mathrm{H}_{\mathrm{o}}=0.10$.

Figure 8. Effect of summation interval $(\Delta \mathrm{X})$ for $\Delta \mathrm{H}_{\mathrm{o}}=4$. 

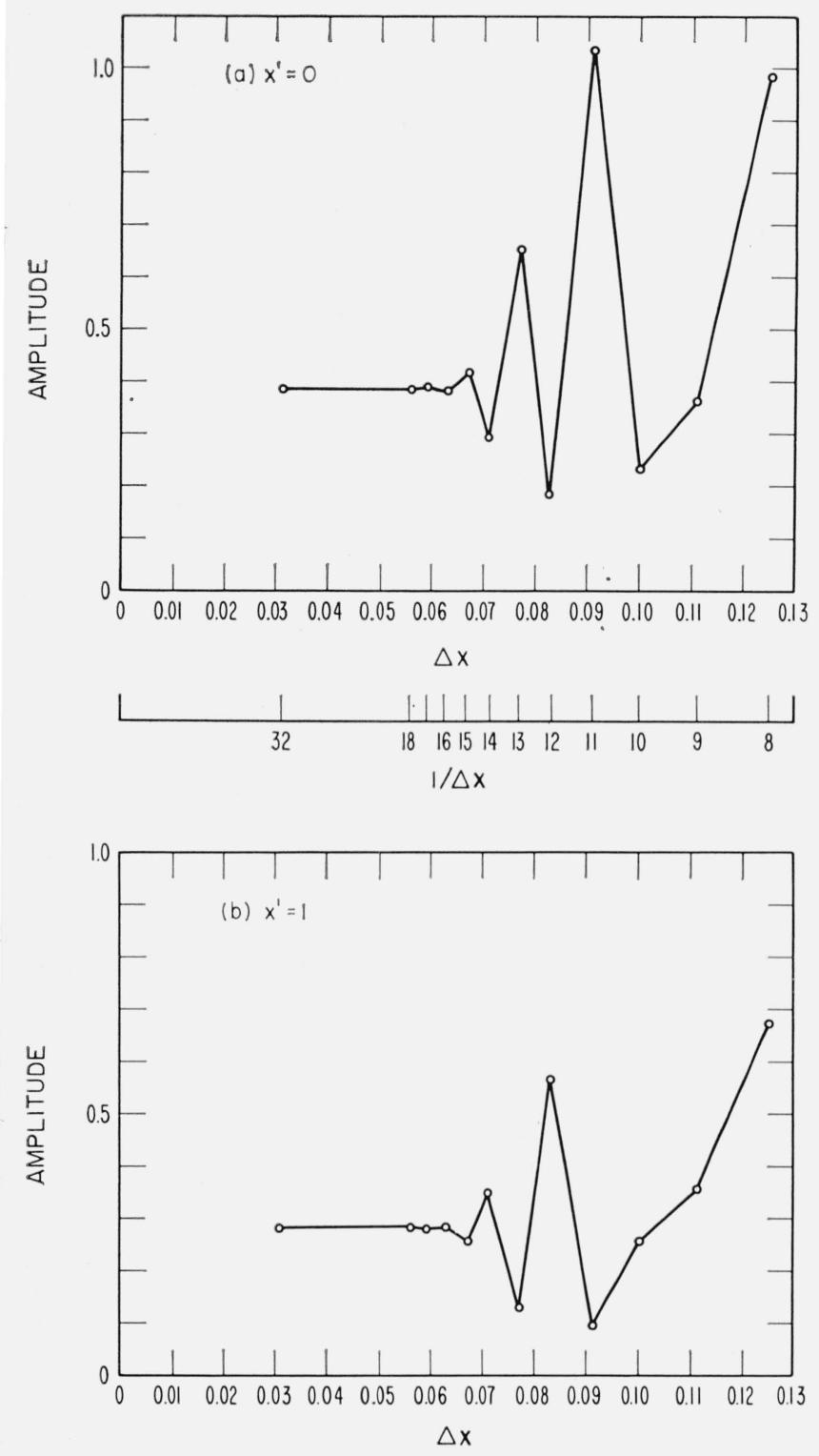

Figure 9. Effect of summation interval $(\Delta \mathrm{X})$ on computed amplitude.

both intervals are satisfactory. According to these tests the interval can be so large that the phase fluctuation in the diffraction screen is permitted to change by several radians between sample points. This, and the need to specify the shape of the modulation in sufficient detail, are the criteria to be kept in mind when deciding what summation interval should be used in a particular instance.

\subsection{Discussion}

The numerical method appears to be accurate in computing diffraction from both shallow and deep sinusoidal phase screens. It is estimated that on an IBM 7090 computer the calculation for $H=20$ using a summation interval of $1 / 16$ would take 0.21 min; so even deep-modulation problems can be evaluated quickly and cheaply. The diffraction screens which arise in nature may not normally be sinusoidal in form; nevertheless, as an approximation it is sometimes convenient to consider them so, and the methods outlined so far may be useful in this way.

\section{Random Screens}

\subsection{Simulation of Random Screens}

The diffractio nscreens appropriate to the natural phenomena of radio propagation are usually random in the sense that it is not possible to predict their space and time characteristics in detail. It is usually assumed, though, that their statistical properties may be exactly defined by parameters such as the standard deviation and the autocorrelation function-that is, that the screens are statistically stationary. We will specify a random phase screen by

$$
\sigma(\Delta H)=\sqrt{\overline{(\Delta H)^{2}}}
$$

and

$$
\rho_{m}(\Delta H)=\frac{{\overline{(\Delta H)_{n}(\Delta H)}}_{n+m},}{\sigma^{2}(\Delta H)}
$$

$\sigma$ being the standard deviation and $\rho_{m}$ the correlation coefficient with respect to interval $m$. It is now necessary to generate a set of numbers having these statistical properties, which will represent the variation of $\Delta H$ across the screen.

A series of correlated random numbers, $a$, may conveniently be generated from a series of uncorrelated random numbers, $b$, by

$$
a_{n+1}=f a_{n}+g b,
$$

where $f$ and $g$ are coefficients. If the standard deviation of the $a$ 's is to equal that of the $b$ 's,

$$
g=\sqrt{1-f^{2}} .
$$

Then the $m$ th correlation coefficient is given by

$$
\rho_{m}=f^{m} .
$$

This simple form will be used in the present work; others could be devised if required. Figure 10 shows the values of $\rho_{m}$ computed from 500 or 1000 numbers generated by this series for three values of $f$. They are seen to agree closely with $f^{m}$.

\subsection{Procedure}

Since the numbers are random it is necessary to use enough of them when evaluating their statistical properties. As a guide we note (as can easily be proved) that if the correlation coefficient is of exponential form,

$$
\rho_{m}=\exp \left(-m / m_{0}\right),
$$

then $r$ points of the correlated series contain the same 


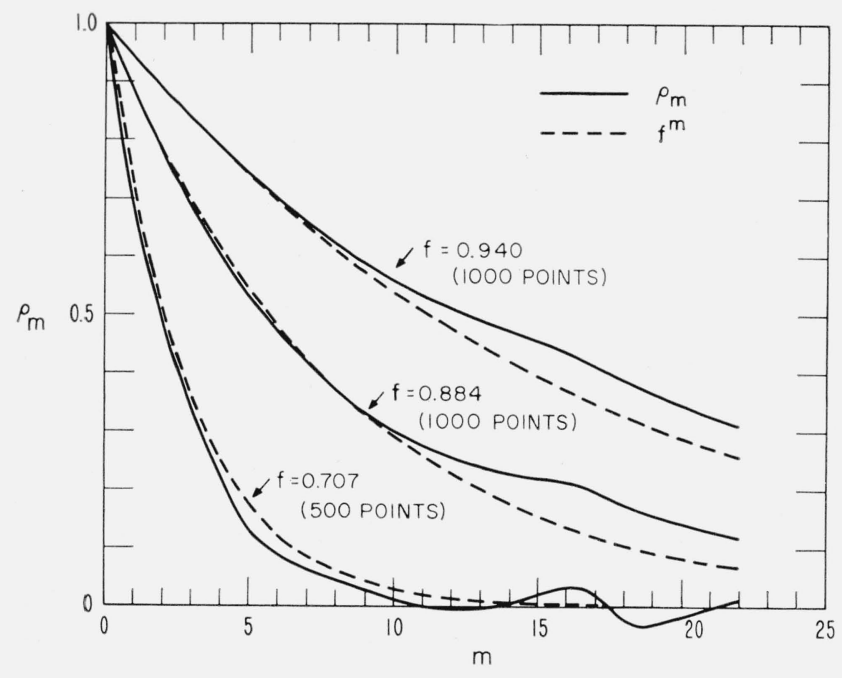

Figure 10. Autocorrelation of generated random screens.

information as $r / 2 m_{0}$ independent points. For smal values of $\rho$, its standard deviation when calculated from $N$ independent points is

$$
\sigma(\rho)=\frac{1}{\sqrt{N-3}} .
$$

We therefore expect the accuracy of an estimate of $\rho$ to be of the order of $\frac{1}{r}$, where $r$ is the num-

$$
\sqrt{\frac{r}{2 m_{0}}-3}
$$

ber of correlated points used to evaluate $\rho$, and $m_{0}$ is the interval at which $\rho$ has fallen to about $1 / e(=0.37)$. Table 1 shows $m_{0}$ and $\sigma(\rho)$ appropriate to 1000 points, for several values of $f$ to be used in computations. The value of $Q$, defined as the distance in wavelengths at which $\rho$ falls to 0.5 , is also given assuming $\Delta X=1 / 8$.

TABle I. Properties of correlated random numbers

\begin{tabular}{c|r|c|c}
\hline \hline$f$ & $m_{0}$ & $\sigma(\rho)($ for $r=1000)$ & $O($ if $\Delta X=1 / 8)$ \\
\cline { 3 - 4 } 0.940 & 16.1 & 0.19 & 1.44 \\
.884 & 8.1 & .13 & 0.728 \\
.794 & 4.3 & .09 & .390 \\
.707 & 2.9 & .08 & .258 \\
.500 & 1.4 & .05 & .125 \\
\hline
\end{tabular}

In the following computations a series representing $\Delta H$ is generated from (8), appropriate values for $f$ and $\sigma(b)$ having been chosen in advance. The properties $\sigma(\Delta H)$ and $\rho(\Delta H)$ are computed for this series. The series $\Delta H$ is then supposed to represent the phase fluctuation sampled at intervals $\Delta X$ along the diffraction plane, and the amplitude $M$ of the diffracted wave is computed at intervals $\Delta X^{\prime}$ (where $\Delta X^{\prime}$ can be equal to $\Delta X, 2 \Delta X$, etc.) along the observation plane. The computation is restricted to amplitude for the sake of brevity, this being the quantity most often observed experimentally. $M$ is evaluated at 500 or 1000 intervals and, finally, the statistical properties $\sigma(M)$ and $\rho(M)$ are computed for comparison with $\sigma(\Delta H)$ and $\rho(\Delta H)$. The distance in wavelengths at which $\rho(M)$ falls to 0.5 is defined as $q$.

It is verified that the interval $\Delta X$ is sufficiently small by repeating the computation with every other point of the series $\Delta H$ eliminated and with $\Delta X$ doubled. With satisfactory intervals there is no significant difference between the two computations. The intervals $\Delta X=1 / 8$ and $\Delta X=1 / 4$ were always found to be adequate with shallow modulation, as would indeed be anticipated from the foregoing sinewave results.

\subsection{Shallow Modulation}

To illustrate the application of the numerical method to shallow random screens, some problems will be explored which lie on the borders of approximations commonly applied in radio diffraction theory. It is generally accepted, for instance, that irregularities much less than a wavelength across $(Q<<1)$ do not propagate and therefore do not appear in the diffracted signal. For irregularities larger than a wavelength it is well known that the statistical form of the diffracted signal becomes identical to that of the diffraction screen when the first Fresnel zone over the diffraction screen is so large that it includes many irregularities, i.e., $1<<Q<<\sqrt{H}$. It is not always evident, however, at what point such approximations become valid. As conditions like these have no special significance in the present numerical method, but also present no particular difficulty, we shall use it to investigate

(i) diffraction from irregularities about one wavelength across: $Q \sim 1$; and

(ii) the transition from the Fresnel to the Fraunhofer region: $Q \sim \sqrt{H}$.

A range of examples has been computed with $Q$ between 0 and 2.7 and with $H$ between 3 and 30 (i. e., $\sqrt{H}$ between 1.7 and 5.5). Typical results are given in figures 11 and 12 which show how the diffracted pattern changes with the distance $(H)$ between the diffraction screen and the observation plane for two values of $Q$. The diffracted amplitude pattern is obviously narrower near the screen, and the amount of fluctuation $(\sigma(M))$ is smaller. Also, the smaller $Q$, the less is the dependence of $q$ on $H$. In figure 12 , where $Q<1$, the correlation function has a different shape at the two planes, which is consistent with the evanescence of irregularities much smaller than a wavelength. Nevertheless a considerable depth of fluctuation remains even at $H=30$. Figure 13 shows the dependence of the pattern width in the observation plane (q) on that in the diffraction plane $(Q)$ at a constant $H=9$. In all cases $\sqrt{H}>Q$. It is seen that $q<Q$ for the larger values but that $q>Q$ for the smaller ones. Overall, $q$ varies much less than $Q$. The depth of fluctuation (expressed as $\left.\frac{\sigma(M)}{\sigma(\Delta H)}\right)$ passes through a maximum near $Q=1$. The loss of fluctuation for $Q>1$ is probably because $Q$ is not sufficiently small compared with the first Fresnel zone, and the loss for $Q<1$ is 

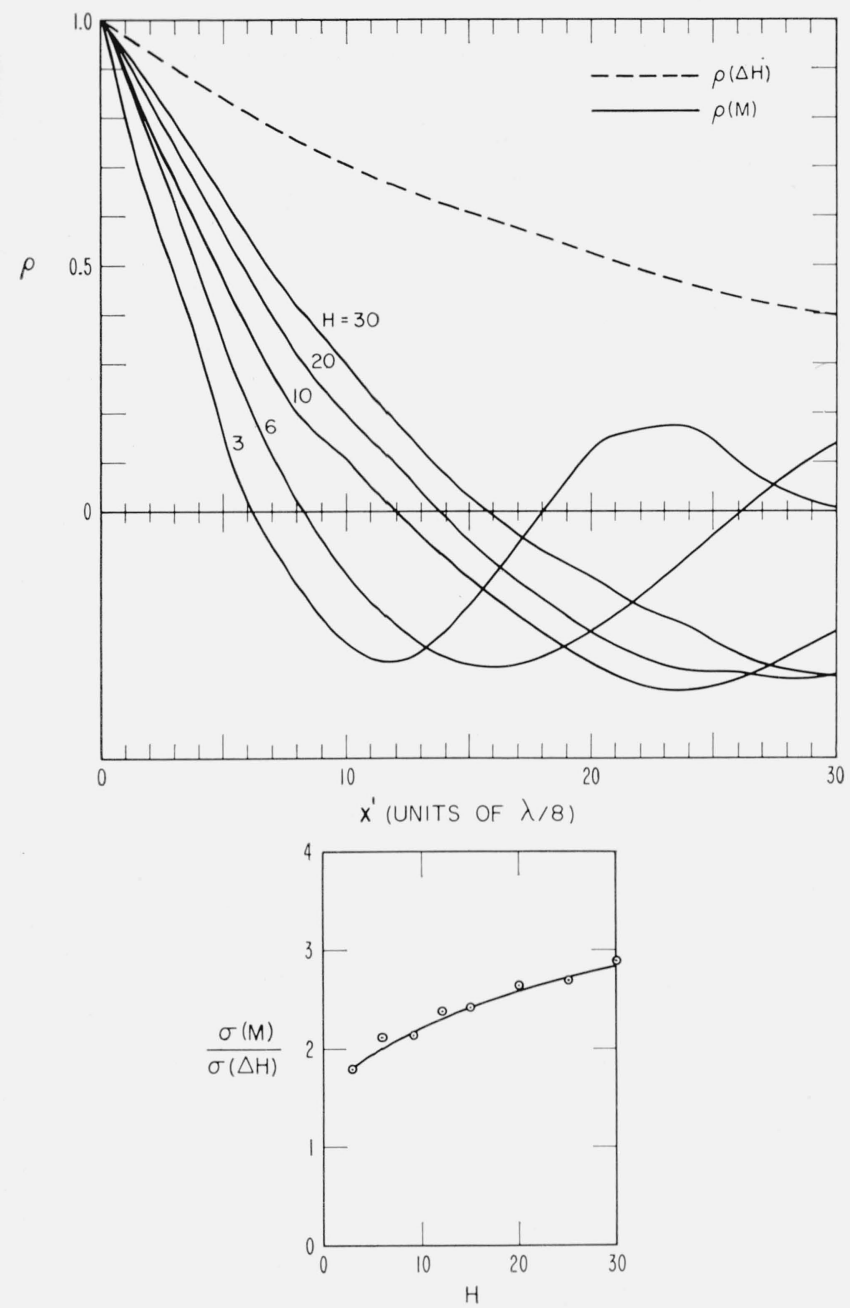

Figure 11. Diffraction patterns for $\mathrm{Q}=2.55$.

probably caused by evanescence of the finer components. Nowhere in these computations does $\frac{\sigma(M)}{\sigma(\Delta H)}$ reach the theoretical limit $\sqrt{2 \pi}(=4.44)$ which is anticipated in the condition $1<<Q<<\sqrt{H}$. It is clear that situations up to $\sqrt{H}=4 Q$ remain well within the Fresnel region, with $\sigma(M)<\sqrt{2} \pi \sigma(\Delta H)$ and $q<Q$.

A particularly interesting case is that for $Q=0$. In contrast to previous work [Bowhill, 1957; Ratcliffe, 1956], the present calculations give $q>0$, $\sigma(M)>0$. This case has been computed for a range of $H$ between 3 and 48 , the calculations being performed with two different sets of random numbers. The results are given in table 2 . There is no apparent trend with $H$, differences being attributed to statistical error. The mean of thesa curves is plotted in figure 14. The existence at $Q=0$ of a finite $q$ of about $\lambda / 4$, and of a residual $\sigma(M)$ o about $2 \sigma(\Delta H)$, is probably due to contributions from signals scattered obliquely; these will obviously be correlated for displacements $X^{\prime}$ up to about $\lambda / 4$. Theoretical treatments that presume a small-angle approximation will miss these contributions.
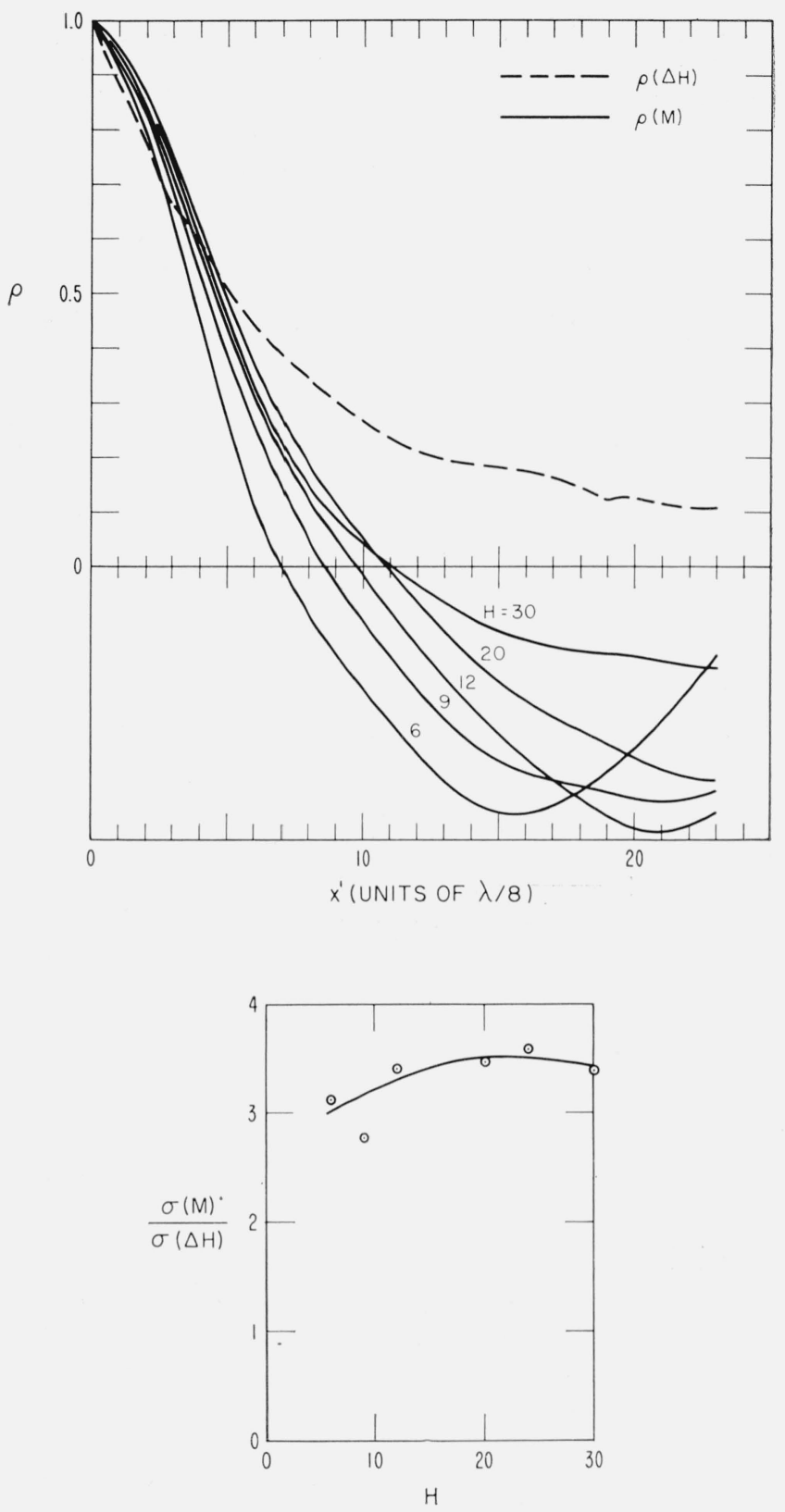

Figure 12. Diffraction patterns for $\mathrm{Q}=0.625$.

Table 2. Computations for $\mathrm{Q}=0$

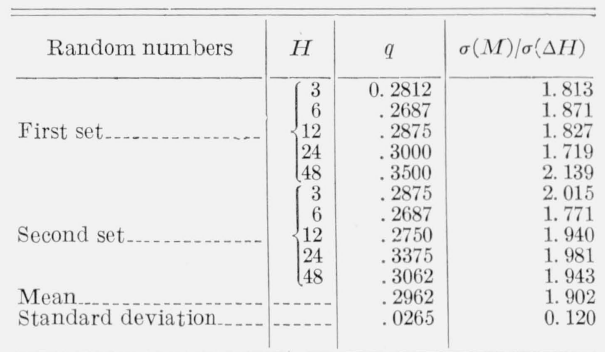

As a summary of the behavior over the full range of computations, figures 15,16 and 17 show the 

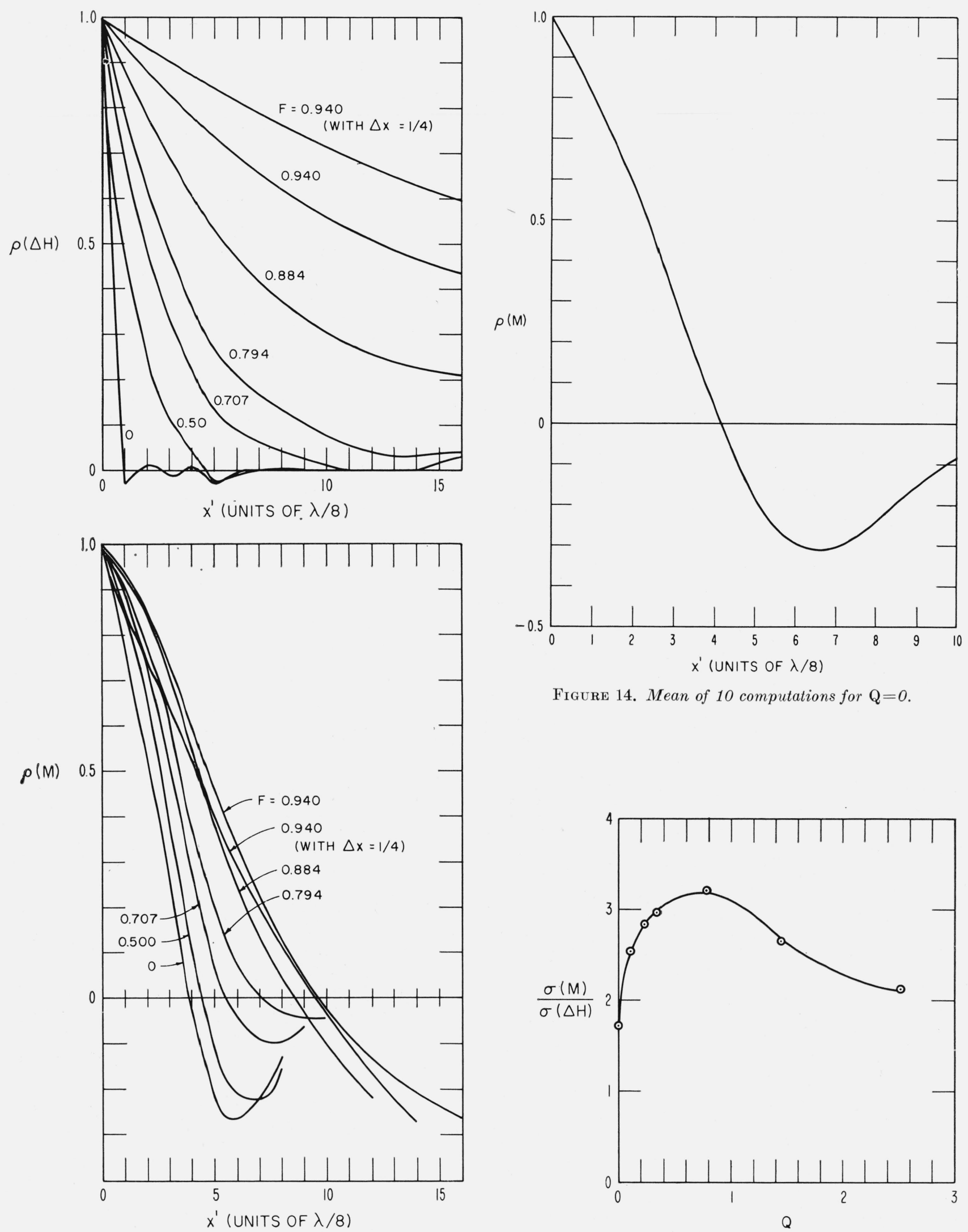

Figure 14. Mean of 10 computations for $\mathrm{Q}=0$.

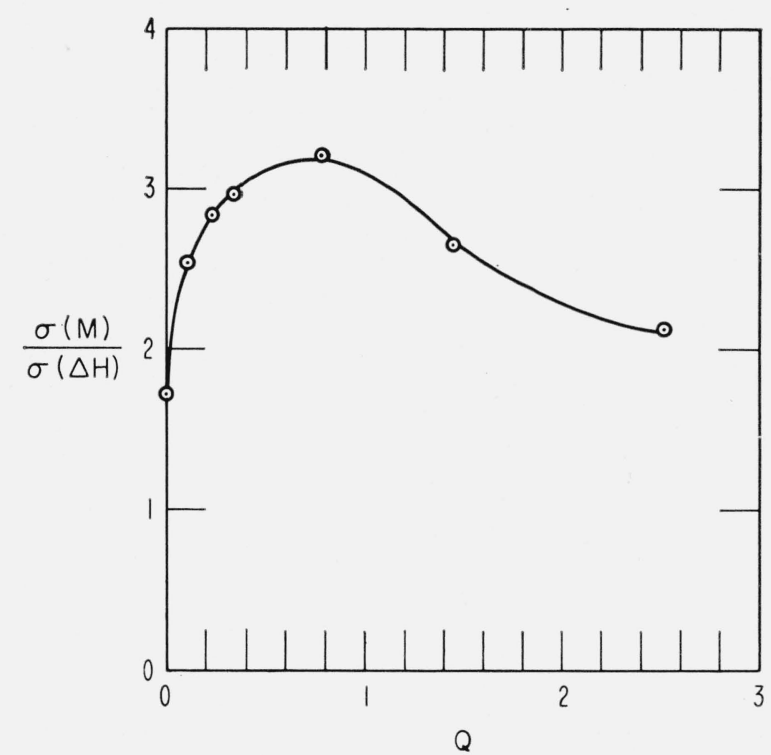

Figure 13. Diffraction patterns for $\mathrm{H}=9$. 

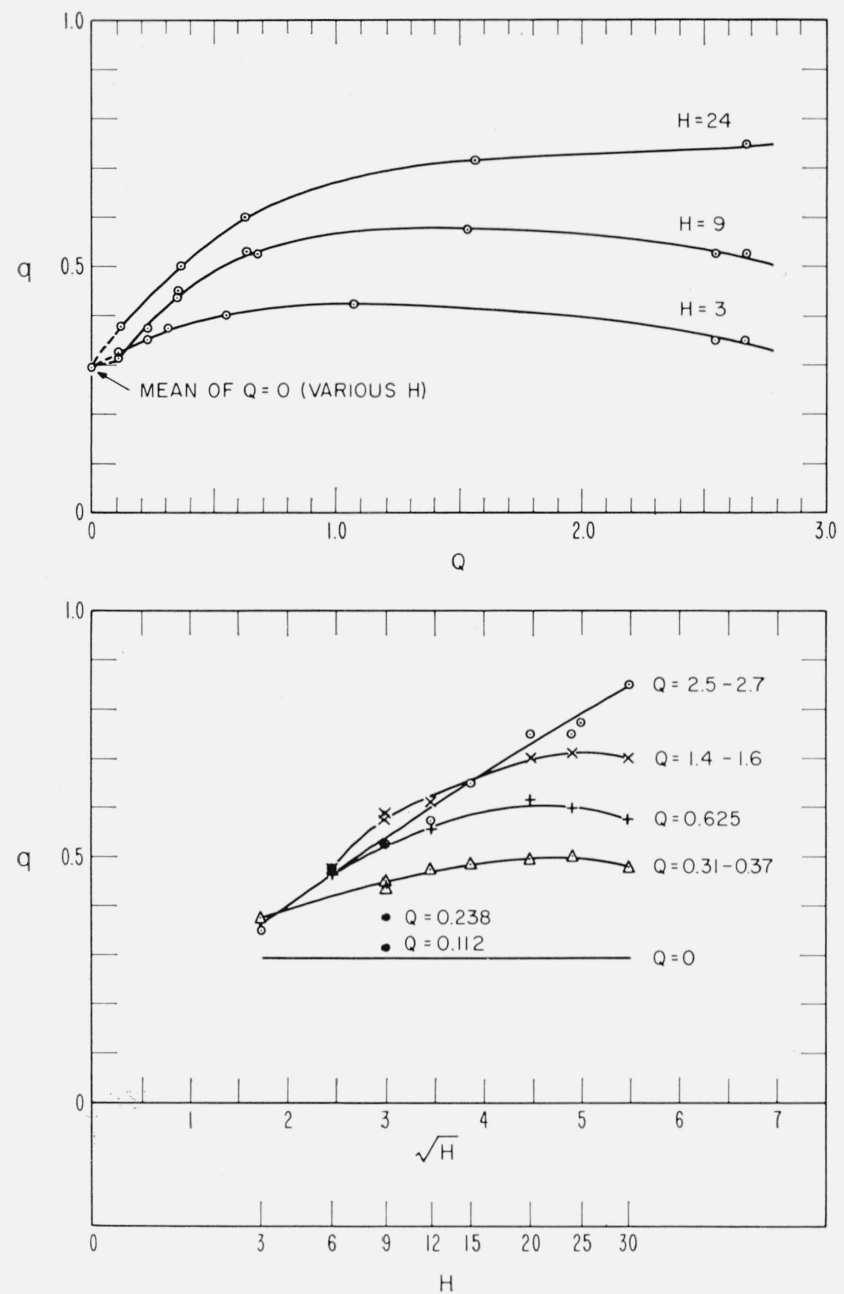

FiguRE 15. Shallowly modulated random screen: width of diffracted amplitude pattern.

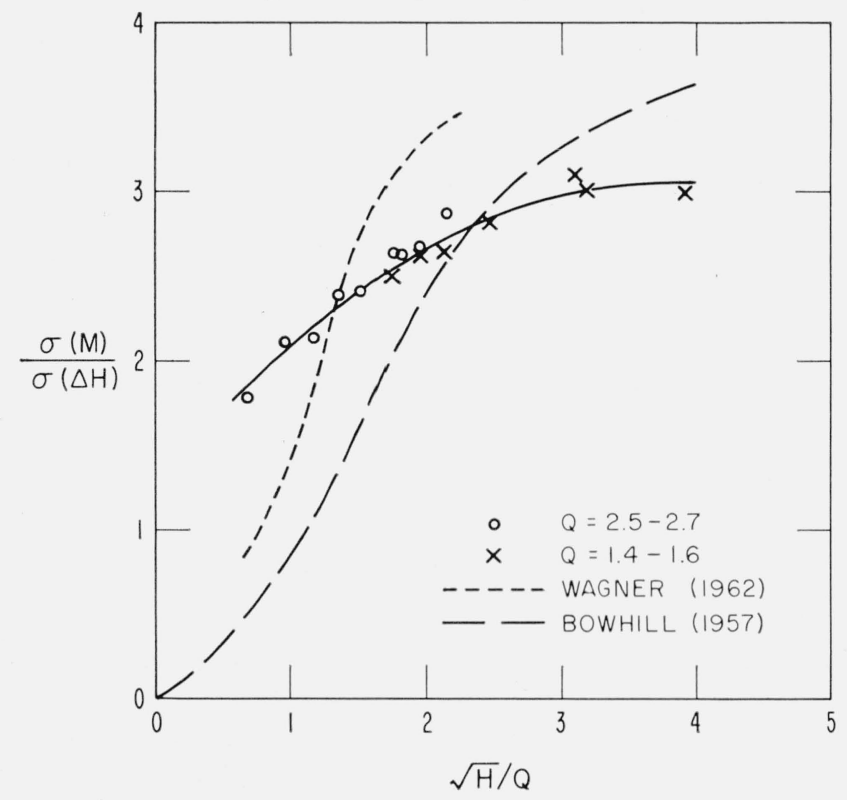

Figure 16. Change of amplitude fluctuation with $\sqrt{\mathrm{H}} / \phi$.
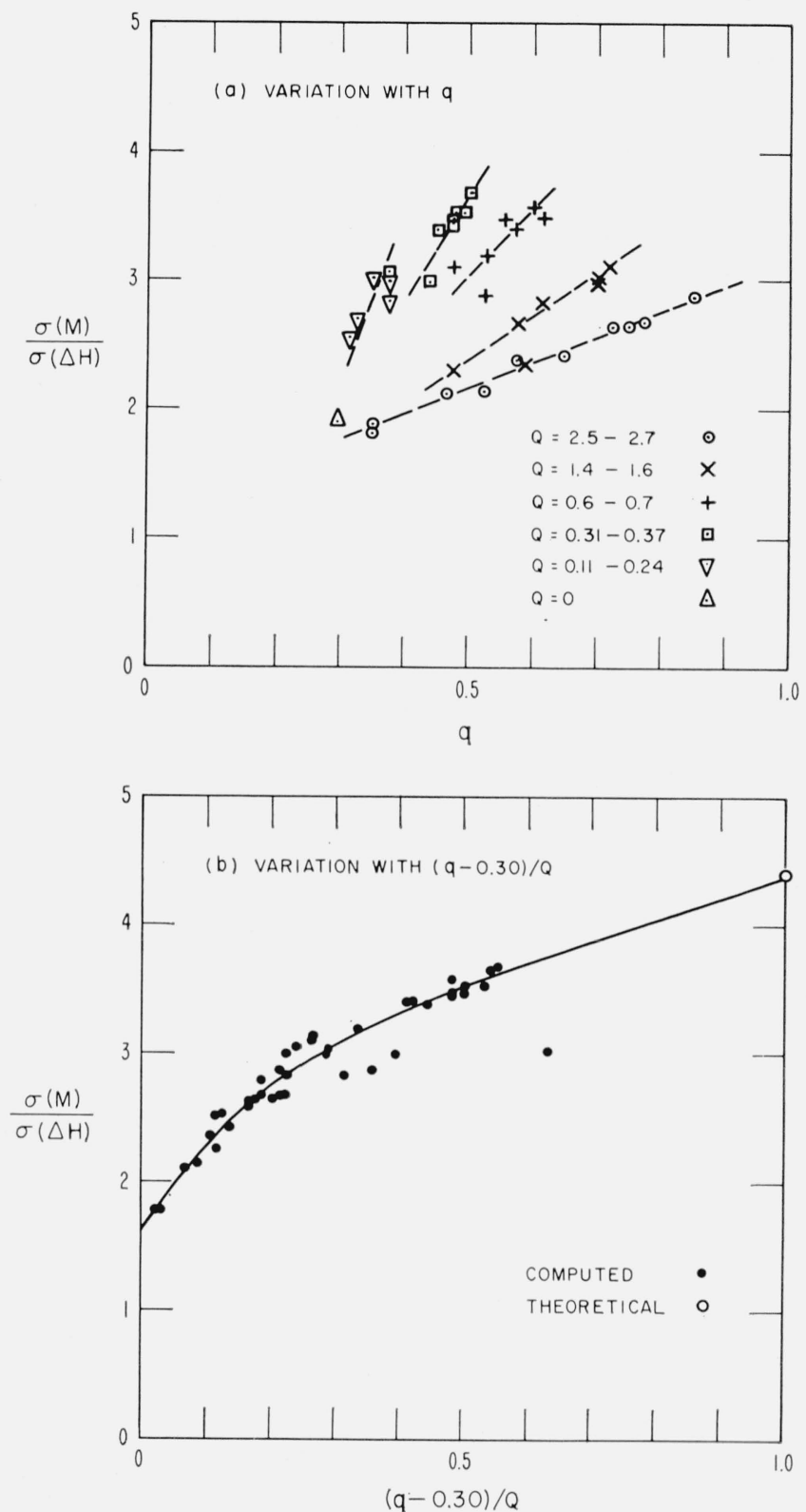

Figure 17. $\sigma(\mathrm{M}) / \sigma(\Delta \mathrm{H})$ for shallow modulation.

width of the amplitude pattern $(q)$ and the depth of amplitude fluctuation $(\sigma(M))$. Figure 15 illustrates the behavior of $q$ :

(i) If $Q<1, q$ increases with increase of $Q$. However, it changes relatively slightly with $H$, passing, in fact, through a maximum.

(ii) If $Q>1, q$ changes more slowly with $Q$ but depends more strongly on $H$. It appears that $q \propto \sqrt{H}$, at least to $\sqrt{H}=2 Q$, when $Q=2.5$.

The fluctuation depths computed for $Q>1$ are given in figure 16 as a function of $\sqrt{H} / \mathrm{Q} . \quad \sigma(M)$ is expected to fall off at the smaller values of $\sqrt{H} / \mathrm{Q}$ because irregularities very large compared to the early Fresnel zones will make negligible contribution. By similar reasoning, $\sigma(M)$ must be zero when $\sqrt{H} / \mathrm{Q}$ is zero. 
The slope found here is very different from the theoretical results of Bowhill [1957] and Wagner [1962] which are also plotted. It must be realized, though, that the three examples are for different forms of irregularity: Bowhill's for a Gaussian correlogram falling to 0.61 at $Q$; Wagner's for a "medium cutoff" power spectrum; and the numerical method for a correlogram of form $f^{m}$ falling to 0.50 at $Q$.

It was not possible to find any simple empirical relation that would describe the variation of $\sigma(M)$ in terms of $H$ and $Q$ over the whole range of $Q$. A relation between $\sigma(M) / \sigma(\Delta H)$ and $q$ derived from the same computation is apparent, however. As indicated in figure $17 \mathrm{a}$, the points derived for a given $Q$ tend to lie on a straight line of slope proportional to $1 / Q$ which passes near the point computed for $Q=0$. This rather curious result is further illustrated in figure $17 \mathrm{~b}$, which shows how $\sigma(M) / \sigma(\Delta H)$ is simply related to a quantity $(q-0.30) / Q$. The scatter is probably no more than statistical error, the points which deviate most having been derived from small values of $Q$. For diffraction in the Fraunhofer region $(\sqrt{ } H>>Q)$ it is to be expected that $\sigma(M) / \sigma(\Delta H)=4.44$, and that $q=Q$ (both being large compared to unity) so that $(q-0.30) / Q=1$. The trend of figure $17 \mathrm{~b}$ indeed passes through this point.

In illustrating the use of the numerical method with shallow random screens, the following two results have emerged:
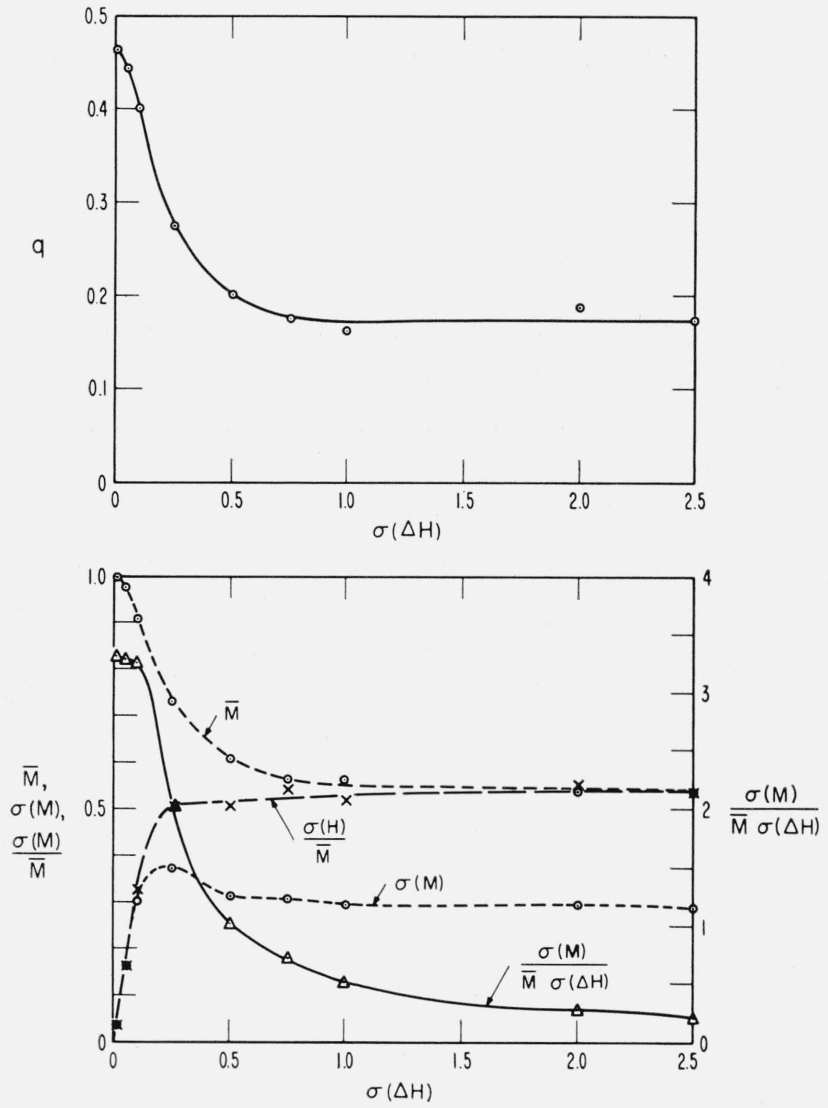

Figure 18. Deep random modulation. (i) Even very small irregularities produce a diffraction pattern with finite fluctuation depth and finite pattern width.

(ii) Fraunhofer diffraction - in which the forms of the diffraction screen and of the amplitude pattern in the observing plane are identical, and in which the relative fluctuation depths are simply relatedcannot be considered to apply until the first Fresnel zone is many times the pattern width.

Such results will have a bearing on the interpretation of experimental data of LF and VLF fading, since a $100 \mathrm{kc} / \mathrm{s}$ signal received at the ground is 30 wavelengths from a diffraction screen at a height of 90 $\mathrm{km}$ and a $15 \mathrm{kc} / \mathrm{s}$ signal is a mere 3.5 wavelengths from a screen at $70 \mathrm{~km}$.

\subsection{Deep Modulation}

To illustrate the method's application to deep random phase screens, one example has been computed which illustrates how the pattern width and depth of amplitude fluctuation change with increasing depth of phase modulation. It is taken that the diffraction and observation planes are nine wavelengths apart, and the phase perturbation is of small horizontal scale: $Q=0.369$. The results are shown in figure 18. The effect of increasing $\sigma(\Delta H)$ is initially to reduce the width of the amplitude pattern, to reduce the mean amplitude, and to increase the fluctuation depth. Beyond $\sigma(\Delta H)=1$, however, these quantities are virtually independent of modulation depth. Presumably, additional contributions to the fading now go into components with structure too small for them to propagate a distance of nine wavelengths. An additional point of interest is that $\sigma(M)=M^{2}$ when $\sigma(\Delta H)$ is large. The interval $\Delta X=$ $1 / 8$ was found to be adequately small in all these computations.

\subsection{Discussion}

Because of the need to obtain an adequate statistical sample, computation with random screens is much lengthier than it is with sinusoidal ones. For instance, the computation takes $11.6 \mathrm{~min}$ in the case $H=20, \Delta X=1 / 8$, when 1000 amplitude points are taken. A limited amount of random screen computation is probably justified, however, because of the closer approach to experimental reality. A set of computations, such as those for shallow modulation, can clearly be applied to practical propagation studies. If it seems reasonable to assume an initial modulation of phase only, and if the present form of correlation coefficient is taken, then figure 15 relates $q, Q$, and $H$. An observation of $q$ and an estimate of $H$ then lead to $Q$. Having measured $\sigma(M)$ it is then possible, from figure 17 , to determine $\sigma(\Delta H)$. It may also be possible to find $\sigma(\Delta H)$ from $\sigma(M)$ and $\sigma(\phi)$ - though this has not been done here. In that event one could work back to a determination of $H$.

A number of improvements and extensions are possible. Computational refinement may speed up the computer program (whose block diagram is given 
in appendix 1). It would be logical to make computations for different forms of correlation coefficient, for a point source, and for oblique-incidence propagation. The model could conceivably be extended to two-dimensional (or even three-dimensional) irregularities. Finally, it may be advantageous to evaluate the phase as well as the amplitude properties of the diffracted waves.

\section{Conclusions}

The object of this work has been to investigate a proposed numerical technique for solving diffraction problems. The technique in its present form appears to be appropriate for radio problems where the irregularities in the diffraction screen are a few wavelengths across and where the observation plane is not more than a few tens of wavelengths away. These are conditions of practical concern in the ionospheric propagation of LF and VLF signals. It has been shown that the technique gives accurate results with sinewave modulation across the screen, and it has been found that, even for deep modulation, integration intervals can be larger than might be thought at first sight. It has been possible to derive original results for random screens observed in the Fresnel region.

Although the numerical method is not always the best one to use, and is limited by considerations of computing time, there are cases where it is very convenient and it may be particularly valuable in the interpretation of experimental diffraction data.

\section{References}

Booker, H. G., J. A. Rateliffe, and D. H. Shinn (1950), Diffraction from an irregular screen with applications to ionospheric problems, Phil. Trans. Roy. Soc. A 242, 579.

Born, M., and E. Wolf (1959), Principles of Optics, 379 (Pergamon Press, New York and London).

Bowhill, S. A. (1957), Ionospheric irregularities causing random fading of very low frequencies, J. Atmospheric Terrest. Phys. 11, 91.

If all $\Delta H$ used

\section{$\downarrow$}

Generate random number $(B)$ with

standard deviation $\sigma_{r}(\Delta H)$

Compute $\Delta H_{n+1}=F \Delta H_{n}+G B$

repeat for $\left(N_{T}+50\right)$

Store $\Delta H$ except for first 50 values ${ }^{(11)}$
Bowhill, S. A. (1961), Statistics of a radio wave diffracted by a random ionosphere, J. Res. NBS 65D (Radio Prop.) No. 3, 275.

Hewish, A. (1951), The diffraction of radio waves in passing through a phase-changing ionosphere, Proc. Roy. Soc. A209, 81 .

Mercier, R. P. (1959), The propagation of fading waves, Phil. Mag. 4, 763.

Mercier, R. P. (1962), Diffraction by a screen causing large random phase fluctuations, Proc. Cambridge Phil. Soc. 58, 382 .

Ratcliffe, J. A. (1956), Some aspects of diffraction theory and their application to the ionosphere, Rept. Progr. Phys. 19, 188.

Wagner, L. S. (1962), Diffraction by a thin phase-changing ionosphere layer, with applications to radio star scintillation, J. Geophys. Res. 67, 4195.

\section{Appendix 1. Block diagram of computer program}

Read basic parameters

$H,^{(1)} 1 / \Delta X,{ }^{(2)} X_{L} / H,{ }^{(3)} \Delta X^{\prime} / \Delta X,{ }^{(4)} F^{(5)}$.

Required standard deviation of $\Delta H,{ }^{(6)}=\sigma_{r}(\Delta H)$.

Number of values of $M^{(7)}$ to be computed $=N_{M}$.

Number of autocorrelation coefficients, $\rho$, to be evaluated for $\Delta H$ and for $M$, and interval between successive values.

Whether or not every other value of $\Delta H$ is to be omitted. ${ }^{(8)}$

Starting parameter for random number generator.

Compute

$$
\begin{aligned}
& \Delta X / H, \Delta X / \sqrt{H} . \\
& N_{c}^{(9)}=\left(1+X_{L} / \Delta X+1 / 2 \Delta X\right) \\
& N_{T}^{(10)}=\left(2 N_{c}-1\right)+\frac{\Delta X^{\prime}}{\Delta X}\left(N_{M}-1\right) \\
& G=\sqrt{1-F^{2}}
\end{aligned}
$$

Generate random screen

If every other $\Delta H$ used

Generate $B_{1}$

Compute $D=F \Delta H_{n}+G B_{1}$

Generate $B_{2}$

Compute $\Delta H_{n+1}=F D+G B_{2}$

repeat for $\frac{N_{T}+50}{2}$

Store $\Delta H$ except for first 25 points ${ }^{(11)}$ 
Find statistical properties of $\Delta H$

Compute mean, $\overline{\Delta H}$

standard deviation, $\sigma(\Delta H)$

autocorrelation coefficients, $\rho(\Delta H)$.

Output these.

Compute

$1 / S e^{(12)},(H S e) / 2 S e^{3 / 2}, H S e$, for $\frac{X}{H}=0\left(\frac{\Delta X}{H}\right) \frac{X_{L}+\frac{1}{2}}{H}$

Generate

sine and cosine tables $0\left(3^{\circ}\right) 357^{\circ}$ and store

Compute

$$
\begin{gathered}
U_{1}=\sum \frac{1+S e}{2 S e^{3 / 2}} \cos 2 \pi\left(H S e+\frac{\Delta H}{S e}\right) ; U_{2}=\sum \frac{1+S e}{2 S e^{3 / 2}} \sin 2 \pi\left(H S e+\frac{\Delta H}{S e}\right) \\
\text { for } \frac{X}{H}=\frac{-X_{L}}{H}\left(\frac{\Delta X}{H}\right) \frac{+X_{L}}{H} \\
\delta U_{1}^{(13)}=\sum \frac{1+S e}{2 S e^{3 / 2}} \cos 2 \pi\left(H S e+\frac{\Delta H}{S e}\right) ; \delta U_{2}=\sum \frac{1+S e}{2 S e^{3 / 2}} \sin 2 \pi\left(H S e+\frac{\Delta H}{S e}\right) \\
\text { for } \frac{X}{H}=\frac{X_{L}}{H}\left(\frac{\Delta X}{H}\right) \frac{X_{L}+\frac{1}{2}}{H} \\
M=\frac{\Delta X}{\sqrt{H}} \sqrt{\left(U_{1}+\delta U_{1}\right)^{2}+\left(U_{2}+\delta U_{2}\right)^{2}}
\end{gathered}
$$

Shift

values of $\Delta H$ by $\frac{\Delta X^{\prime}}{\Delta X}$ intervals.

Repeat for $N_{M}$

Find statistical properties of $M$

Compute mean, $\bar{M}$, standard deviation, $\sigma(M)$

autocorrelation coefficients, $\rho(M)$.

Output these

Output next random number parameter. ${ }^{(14)}$

$1 H$ is the distance in wavelengths between diffraction and observation planes.

${ }^{2} \Delta X$ is the distance in wavelengths between sample points in the diffraction

plane

$X_{L}$ is the limit of summation in the diffraction plane.

$4 \Delta X^{\prime}$ is the distance in wavelengths between points in the observation plane at which results are to be computed.

${ }_{5} \mathrm{~F}$ is the parameter determining the width of the correlation pattern at the diffraction screen.

$\Delta H$ is the phase deviation.

$7 M$ is the amplitude at the observation plane.

8 Alternate values of $\Delta H$ can be omitted to test whether $\Delta X$ is sufficiently small.

${ }^{\circ} N_{c}$ is the number of values of $|X|$ required.

${ }_{10} N_{T}$ is the total number of diffraction screen samples required.

11 Early points are dropped to ensure statistical uniformity in the series.

$12 S e=\sqrt{1+\frac{X^{2}}{H^{2}}}$

$13 \delta U_{1}, \delta U_{2}$ are the correction terms for finite $X_{L}$.

${ }_{14} \delta U_{1}, \delta U_{2}$ are the correction terms for finite $X_{L}$.
The next random number parameter is saved so that computations can be repeated with an independent set of numbers.

Note: The program is in Fortran. Tape storage is used for extra capacity. Assuming $N_{M}=1000, N_{c}$ can be up to 4000 . 